La revue La revue pour l'histoire du CNRS

POUR L LHISTOIRE DU CNRS $3 \mid 2000$

Regards sur l'étranger

La «structure fine » de la relativité restreinte

Yves Pierseaux, L'Harmattan, Paris, 1999

Marc Raunni

(2) OpenEdition

Journals

Édition électronique

URL : https://journals.openedition.org/histoire-cnrs/383

DOI : $10.4000 /$ histoire-cnrs.383

ISSN : 1955-2408

Éditeur

CNRS Éditions

Édition imprimée

Date de publication : 3 novembre 2000

ISBN : 978-2-271-05786-0

ISSN : $1298-9800$

Référence électronique

Marc Raunni, «La « structure fine » de la relativité restreinte », La revue pour l'histoire du CNRS [En ligne], 3 | 2000, mis en ligne le 06 mars 2006, consulté le 20 mai 2021. URL : http://

journals.openedition.org/histoire-cnrs/383; DOI : https://doi.org/10.4000/histoire-cnrs.383

Ce document a été généré automatiquement le 20 mai 2021.

Comité pour I'histoire du CNRS 


\section{La « structure fine » de la relativité restreinte}

Yves Pierseaux, L'Harmattan, Paris, 1999

Marc Raunni

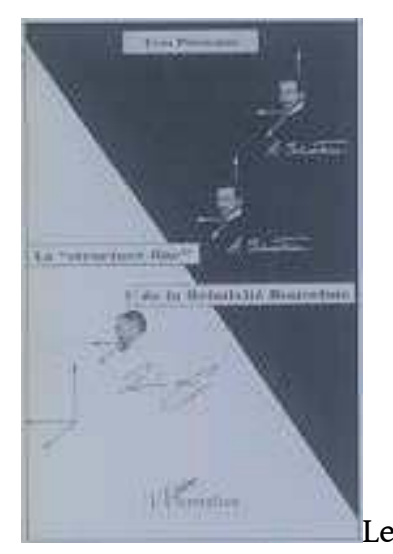

Les cas de découvertes simultanées intriguent depuis longtemps les historiens pour la simple raison qu'à l'identité ou presque des résultats on peut opposer la diversité des contextes socioculturels. Il s'agit de cas privilégiés où la place $\mathrm{du}$ contexte peut être explorée par rapport au «déterminisme» de la culture scientifique au niveau international. C'est aussi une occasion de relativiser l'aspect formel des résultats scientifiques qui prennent toute leur valeur si on les lit insérés dans un contexte culturel. La relativité restreinte est l'un de ces cas. L'intérêt de son étude repose aussi sur la personnalité des deux protagonistes principaux: Albert Einstein et Henri Poincaré. Leurs travaux ont été publiés la même année à quelques mois d'intervalle et, fort probablement, sans qu'A. Einstein ait eu connaissance de l'article de H. Poincaré. À l'époque (1905), H. Poincaré est au sommet de sa renommée, alors qu'A. Einstein est un jeune employé de l'office des brevets suisses.

1 Étant donné la personnalité des deux savants, on s'est très tôt intéressé à départager leurs différences et ce que l'on devait à l'un et à l'autre. Cela a commencé dans le climat nationaliste de l'après Première Guerre mondiale. Le jeune Wolfgang Pauli rédige à la demande d'Arnold Sommerfeld pour l'Encyclopédie des mathématiques un article 
historique où il s'efforce, à la demande expresse du commanditaire, d'attribuer à chacun des protagonistes la part qui leur était due. De même, E. Guillaume, Suisse d'origine, dirigeait à Paris le Bureau international des poids et mesures. Dans ce climat, fit scandale, en Allemagne comme en France, la déclaration d'A. Einstein affichant son admiration pour H. Poincaré. Depuis, le nombre d'auteurs, historiens ou scientifiques ayant repris cette question est fort élevé. C'est dire la difficulté relevée par Y. Pierseaux en choisissant ce sujet de thèse. D'ailleurs l'abondante bibliographie prouve bien que l'auteur se situe dans la continuité d'un débat déjà riche.

2 Formé à la philosophie et à la physique, Y. Pierseaux décortique avec une minutie de micro chirurgien les articles fondateurs. Il entre dans les aspects techniques et cherche, à partir de là, à saisir le contexte scientifique auquel ils font référence. Il faut être en mesure de suivre la technicité de l'argumentation de l'auteur pour apprécier ce travail en profondeur. Ce travail est d'autant plus indispensable que l'auteur refuse de faire sienne la justification, souvent répétée, que les différences entre $\mathrm{H}$. Poincaré et $\mathrm{A}$. Einstein seraient dues au fait que le premier était plus mathématicien que physicien, ce qui serait exactement l'inverse pour le deuxième. Cette explication, il est vrai, revient à banaliser l'analyse historique et à appauvrir la personnalité des deux savants. Il est donc essentiel de prendre en compte les personnalités de l'époque avec lesquelles ils dialoguaient comme Hendrik Lorentz pour H. Poincaré (théorie de l'électron et expériences de Michelson et Morley) et Ludwig Boltzmann pour A. Einstein (mécanique statistique). Le dialogue avec $\mathrm{H}$. Lorentz, personnage clé de la physique théorique de l'époque, couronné du prix Nobel en 1902, est fondamental pour comprendre l'attitude d'H. Poincaré et sa "discrétion» sur l'existence de l'éther. Sur cette question, H. Poincaré s'était déjà exprimé dans son Traité d'optique, en la définissant comme une question de métaphysique plus que de physique. De même, la référence à L. Boltzmann est capitale si l'on veut saisir l'importance de l'invariance des lois physiques qui préoccupe A. Einstein. Ce simple rappel donne une idée de la reconstruction faite par Y. Pierseaux de ces «dialogues" créatifs. Je regrette l'absence d'un personnage: Pierre Curie. Certes, en 1905, il est occupé par les travaux sur le radium et il disparaît quelques temps après. Néanmoins, il a introduit en physique l'idée selon laquelle les règles de symétrie sont indispensables pour comprendre la manifestation de phénomènes physiques. La démonstration d'H. Poincaré affirmant que les transformations d'H. Lorentz forment un groupe est alors une manière "élégante» de prouver ce que $\mathrm{H}$. Lorentz avait réussi « avec des coups de pouce » : le résultat négatif des expériences de Michelson-Morley. Cette remarque ne doit pas diminuer l'intérêt que présente ce livre.

\section{AUTEUR}

MARC RAUNNI

Historien 